Series A

I. MATHEMATICA

549

\title{
DIE LOGARITHMISCHE METHODE \\ IN DER WERTVERTEILUNGSTHEORIE PSEUDOANALYTISCHER FUNKTIONEN
}

YoN

HEINRICH BEGEHR

Herrn Professor In. Alexander Dinghas zu seinem 65. Cehnt-tage widmet

H ELSIN K I 1973

S U O M A L I NEN TIEDEAKATEMIA

https://doi.org/10.5186/aasfm.1973.549 


\section{Copyright (C) 1973 by \\ Academia Scientiarum Fennica \\ ISSN 0066-1953 \\ ISBN 951-41-0124-3}

Am 12. Februar 1973 vorgelegt von R. Nevanlinna 


\section{Die logarithmische Methode in der Wertverteilungstheorie pseudoanalytischer Funktionen}

Der ursprüngliche Beweis des zweiten Hauptsatzes in der Theorie der Wertverteilung meromorpher Funktionen wurde von R. Nevanlinna [7] mit Hilfe der Poisson-Jensenschen Formel durch Abschätzung der logarithmischen Ableitung gegeben. Die auf Grund der Pompeiuschen Formel abgeänderte Poisson-Jensensche Formel erlaubt eine Abschätzung der logarithmischen $(F, G)$-Ableitung einer $(F, G)$-pseudoanalytischen Funktion und damit die Anwendung der logarithmischen Methode für pseudoanalytische Funktionen erster Art. Eine Wertverteilungstheorie für diese Funktionenklasse hat Habetha [4] unter Verwendung der potentialtheoretischen Methode von Ahlfors gegeben, nachdem die entsprechenden Untersuchungen für pseudoanalytische Funktionen zweiter Art von af Hällström [5] und anderen mit den topologischen Hilfsmitteln durchgeführt worden waren, die Ahlfors in seiner Theorie der Überlagerungsflächen entwickelt hat.

1. Pseudoanalytische Funktionen. Die Rolle von 1 und $i$ als Basis für den reellen Vektorraum der komplexen Zahlen $C$ kann von zwei in $C$ definierten komplexen Funktionen $F$ und $G$ übernommen werden, die der Bedingung

$$
0<\operatorname{Im}\{\overline{F(z)} G(z)\} \quad(z \in C)
$$

genügen. Sind $F$ und $G$ in einem Gebiet $D$ von $\hat{C}=C \cup \infty$ gegeben und genügen sie dort der Ungleichung (1), so kann man jede in $D$ definierte komplexe Funktion $w$ mit Hilfe von zwei eindeutig festgelegten reellen Funktionen $\Phi$ und $\Psi$ durch

$$
w(z)=\Phi(z) F(z)+\Psi(z) G(z) \quad(z \in D)
$$

darstellen. Die Klasse der bezüglich eines solchen Funktionenpaares $(F, G)$ in $D$ pseudoanalytischen Funktionen, auch $(F, G)$-pseudoanalytische oder kurz pseudoanalytische Funktionen genannt, wird durch diejenigen komplexen Funktionen gebildet, für die in $D$ die $(F, G)$-Ableitung 


$$
\dot{v}(z)=\frac{d_{(F, G)} w(z)}{d z}=\lim _{\zeta \rightarrow z} \frac{w(\zeta)-\Phi(z) F(\zeta)-\Psi(z) G(\zeta)}{\zeta-z} \quad(z \in D)
$$

existiert, wobei $w$ in $D$ durch (2) dargestellt ist. Zu einer in $D(F, G)$ pseudoanalytischen Funktion $w$ mit der Darstellung (2) heißt

$$
* w(z)=\Phi(z)+i \Psi(z) \quad(\bmod (F, G))
$$

die zugehörige $(F, G)$-pseudoanalytische Funktion zweiter Art. Beide Funktionen, $w$ wie $* w$, hat man als unterschiedliche Darstellungen desselben mathematischen Objektes aufzufassen. Die $(F, G)$-pseudoanalytischen Funktionen bilden einen linearen Raum, der die (erzeugenden) Funktionen $F$ und $G$ enthält. Wegen

$$
\dot{F}(z) \equiv 0, \quad \dot{G}(z) \equiv 0
$$

hat man Funktionen der (und nur der) Form

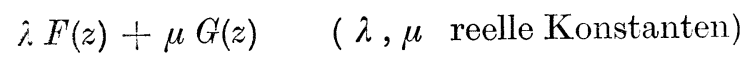

als $(F, G)$-pseudoanalytische Konstanten aufzufassen. Diese Tatsache legt folgende auf Bers [2] zurückgehende Definition nahe. Die pseudoanalytische Funktion $w$ nimmt im Punkt $z_{0}$ die komplexe Zahl a genau dann an, wenn $* w\left(z_{0}\right)=a$ gilt, also wenn $w(z)-\alpha(z)$ mit $* \alpha(z) \equiv a$ in $z_{0}$ eine Nullstelle hat. In der Umgebung von isolierten Singularitäten zeigen pseudoanalytische Funktionen asymptotisch das gleiche Verhalten wie analytische Funktionen. In der Nähe von Null- bzw. Polstellen gelten

$$
\begin{aligned}
& w(z)=c\left(z-z_{0}\right)^{n}(1+o(1)) \\
& \dot{v}(z)=n c\left(z-z_{0}\right)^{n-1}(1+o(1)) \quad\left(z \rightarrow z_{0}\right),
\end{aligned}
$$

wenn $|n|>0$ die Vielfach eit der Stelle angibt. Für wesentliche isolierte Singularitäten gilt der Satz von Casorati-Weierstraß. Die Bedeutung der pseudoanalytischen Funktionen besteht einmal in der Quasikonformität von $* w$ und zum anderen in dem Zusammenhang zu gewissen elliptischen Differentialgleichungssystemen. Sind $F$ und $G$ stetige Funktionen, so folgt aus der Existenz von $\dot{w}$ die stetige partielle Differenzierbarkeit von $\Phi$ und $\Psi$ und zwar gelten mit den Operatoren

$$
\frac{\partial}{\partial z}=\frac{1}{2}\left(\frac{\partial}{\partial x}-i \frac{\partial}{\partial y}\right), \quad \frac{\partial}{\partial \bar{z}}=\frac{1}{2}\left(\frac{\partial}{\partial x}+i \frac{\partial}{\partial y}\right)
$$

die Differentialgleichungen

$$
\Phi_{z} F+\Psi_{z} G=\dot{w}, \quad \Phi_{\bar{z}} F+\Psi_{\bar{z}} G=0 .
$$

Aus der letzten Gleichung folgt 


$$
(* w)_{\bar{z}}=\gamma \overline{(* w)_{z}}, \quad \gamma=-\frac{F+i G}{F-i G} .
$$

Die Funktion $* w$ vermittelt eine innere Abbildung im Sinne von Stoïlow, die mit Ausnahme von isolierten Punkten quasikonform mit der Dilatation

$$
\gamma=\frac{1+|\gamma|^{2}}{1-|\gamma|^{2}}
$$

ist. Sind $F$ und $G$ partiell differenzierbar, so folgt aus der Existenz von $\dot{w}$ die partielle Differenzierbarkeit von $w$ und die Gültigkeit der Gleichungen

$$
w_{\bar{z}}=a w+b \bar{w}, \quad \dot{w}=w_{z}-A w-B \bar{w} .
$$

Die (charakteristischen) Koeffizienten $a, b, A, B$ des erzeugenden Paares $(F, G)$ bestimmen sich eindeutig aus den Gleichungssystemen

$$
\begin{array}{ll}
F_{\bar{z}}=a F+b \bar{F}, & F_{z}=A F+B \bar{F}, \\
G_{\bar{z}}=a G+b \bar{G}, & G_{\tilde{z}}=A G+B \bar{G} .
\end{array}
$$

Die Theorie der pseudoanalytischen Funktionen ist als Funktionentheorie der ersten Gleichung in (4) aufzufassen. Sie ist von Bers [2] und unter Zugrundelegung verallgemeinerter Sobolevscher Ableitungen von Vekua [13] entwickelt worden.

Um die Nevanlinnaschen Hauptsätze für pseudoanalytische Funktionen in einfach zusammenhängenden Gebieten herzuleiten, genügt es wegen der konformen Invarianz der Pseudoanalytizität, ein Normalgebiet der Form

$$
z:<R \leqq+\infty
$$

$\mathrm{zu}$ betrachten. Wir werden in diesem Gebiet existierende erzeugende Paare $(F, G)$ betrachten, die die folgenden Bedingungen erfüllen:

(i) $(F, G)$ ist beschränkt,

$$
|F(z)|+|G(z)| \leqq M<+\infty \quad(|z|<R),
$$

und es gilt

$$
0<\Delta \leqq \operatorname{Im}\{\overline{F(z)} G(z)\} \quad(|z|<R) .
$$

(ii) $F$ und $G$ sind stetig partiell differenzierbar, $\left(F_{\bar{z}}, G_{\bar{z}}\right)$ ist ebenfalls beschränkt, etwa durch $M$ aus (i),

$$
\left|F_{\bar{z}}(z)\right|+\left|G_{\bar{z}}(z)\right| \leqq M<+\infty \quad(|z|<R),
$$

und es gelten $(\zeta=\xi+i \eta)$ 
A. I. $\quad 549$

$$
\begin{aligned}
\sup _{|z|<R} & \frac{1}{\pi} \int_{|\zeta|<R}\left[\left|F_{\bar{\zeta}}(\zeta)\right|+\left|G_{\bar{\zeta}}(\zeta)\right|\right] \frac{d \xi d \eta}{|\zeta-z|}=M_{1}<+\infty \\
& \frac{1}{\pi} \int_{|\zeta|<R}\left[\left|F_{\zeta}(\zeta)\right|+\left|G_{\zeta}(\zeta)\right|\right] \frac{d \xi d \eta}{|\zeta|}<+\infty
\end{aligned}
$$

Nach Nirenberg [10] genügen $F$ und $G$ in jedem Bereich von $|z|<R$ einer Hölderbedingung mit festem Exponenten und einer im wesentlichen nur vom Abstand des Bereiches vom Gebietsrand $|z|=R$ abhängenden Konstanten. Demnach ist für $\varrho<R$

$$
\sup _{|z|<\varrho} \frac{1}{\pi} \int_{|\zeta|<\varrho}[|F(\zeta)-F(z)|+|G(\zeta)-G(z)|] \frac{d \xi d \eta}{|\zeta-z|^{2}}=M_{2}(\varrho)
$$

endlich und mit wachsendem $\varrho$ nicht abnehmend und wird als von endlicher Ordnung vorausgesetzt, d. h. mit

$$
\tau(r)= \begin{cases}\log r \quad, & R=\infty \\ \log \frac{1}{R-r}, & R<\infty\end{cases}
$$

soll

$$
\varlimsup_{r \rightarrow R} \frac{\log M_{2}(r)}{\tau(r)}
$$

endlich sein.

(iii) Die partiellen Ableitungen $F_{\bar{z}}$ und $G_{\bar{z}}$ sind Hölderstetig und

$$
\sup _{|z|<\varrho} \frac{1}{\pi} \int_{|\zeta|<\varrho}\left[\left|F_{\bar{\zeta}}(\zeta)-F_{\bar{z}}(z)\right|+\left|G_{\bar{\zeta}}(\zeta)-G_{\bar{z}}(z)\right|\right] \frac{d \xi d \eta}{|\zeta-z|^{2}}=M_{3}(\varrho)
$$

ist von endlicher Ordnung.

Aus den Voraussetzungen über $M_{2}$ und $M_{3}$ folgt, daß auch

$$
\sup _{|z|<\varrho} \frac{1}{\pi} \int_{|\zeta|<\varrho}[|a(\zeta)-a(z)|+|b(\zeta)-b(z)|] \frac{d \xi d \eta}{|\zeta-z|^{2}}=M_{4}(\varrho)
$$

von endlicher Ordnung ist.

Im Fall $R=\infty$ erfüllen die vollständig normalen erzeugenden Paare (vgl. [2], S. 74) die obigen Bedingungen, für endliches $R$ genügen ihnen z. B. erzeugende Paare, die in einem $|z| \leqq R$ enthaltenden Gebiet definiert sind und dort Hölderstetige partielle Ableitungen nach $\bar{z}$ besitzen (vgl. [2], S. 3). 
2. Die allgemeine Poisson-Jensensche Formel. Erster Hauptsatz. Die Formel von Pompeiu, eine komplexe Form der Greenschen Formel, für eine in einem beschränkten, regulären Gebiet D partiell stetig differenzierbare Funktion $w$, die in

$$
\hat{D}=D \cup \Gamma, \quad \Gamma=\partial D
$$

stetig ist,

$$
\int_{\Gamma} w(\zeta) d \zeta+\int_{D} u(\zeta) d \zeta d \bar{\zeta}=0
$$

führt zu der Darstellung

$$
w(z)=\frac{1}{2 \pi i} \int_{\Gamma} w(\zeta) \frac{d \zeta}{\zeta-z}+\frac{1}{2 \pi i} \int_{D} w_{\bar{\zeta}}(\zeta) \frac{d \zeta d \bar{\zeta}}{\zeta-z} \quad(z \in D) .
$$

Für $z$ aus $C-\hat{D}$ ist die linke Seite dieser Gleichung durch null zu ersetzen. Ist $D$ der Kreis $|z|<\varrho<+\infty$, so gilt wegen

$$
\int_{|\zeta|=\varrho} w(\zeta) \frac{\bar{z}}{\varrho^{2}-\bar{z} \zeta} d \zeta+\int_{|\zeta|<\varrho} w_{\bar{\zeta}}(\zeta) \frac{\bar{z}}{\varrho^{2}-\bar{z} \zeta} d \zeta d \bar{\zeta}=0 \quad(|z|<\varrho)
$$

für $|z|<\varrho$ die Darstellung

$$
w(z)=\frac{1}{2 \pi i} \int_{|\zeta|=\varrho} w(\zeta) \frac{\varrho^{2}-|z|^{2}}{|\zeta-z|^{2}} \frac{d \zeta}{\zeta}+\frac{1}{2 \pi i} \int_{\zeta<0} w_{\zeta}(\zeta) \frac{\varrho^{2}-|z|^{2}}{\varrho^{2}-\bar{z} \zeta \zeta d \bar{\zeta}}
$$

Ist $w(z)$ in $|z|<\varrho$ pseudoanalytisch und hat dort endlich viele Nullstellen $a_{u}$ und endlich viele Pole $b_{v}$, so folgt auf dem üblichen Wege eine Darstellung für $\log |w(z)|$. Dabei kann zugelassen werden, daß auch auf $|z|=\varrho$ endlich viele Nullstellen und Pole liegen.

Poisson-Jensensche Formel.

$$
\begin{aligned}
\log |w(z)|= & \frac{1}{2 \pi i} \int_{\zeta \xi=\varrho} \log |w(\zeta)| \frac{\varrho^{2}-|z|^{2}}{|\zeta-z|^{2}} \frac{d \zeta}{\zeta} \\
& +\operatorname{Re}\left\{\frac{1}{2 \pi i} \int_{|\zeta|<!} \frac{w_{\xi}(\zeta)}{w(\zeta)} \frac{\varrho^{2}-|z|^{2}}{\varrho^{2}-\bar{z} \zeta} \frac{d \zeta-z}{\zeta}\right\} \\
& +\sum_{\left|a_{\mu}\right|<\varrho} \log \left|\frac{\varrho\left(z-a_{\mu}\right)}{\varrho^{2}-\overline{a_{\mu}} z}\right|-\sum_{b_{v} \mid<\varrho} \log \left|\frac{\varrho\left(z-b_{v}\right)}{\varrho^{2}-\overline{b_{\nu} z}}\right| .
\end{aligned}
$$

In den Summen über die Nullstellen und Pole tritt jeder Summand der Vielfachheit der Stelle entsprechend häufig auf. 
Für $z=0$ erhält man die Jensensche Formel, die hier in der modifizierten Form angegeben wird.

\section{Jensensche Formel.}

$$
\begin{aligned}
\log \left|c_{\lambda}\right|= & \frac{1}{2 \pi i} \int_{|\zeta|=\varrho} \log |w(\zeta)| \frac{d \zeta}{\zeta}+\operatorname{Re}\left\{\frac{1}{2 \pi i} \int_{|\zeta|<\varrho} \frac{w_{\bar{\zeta}}(\zeta)}{w(\zeta)} \frac{d \zeta d \bar{\zeta}}{\zeta}\right\} \\
& +\sum_{0<\left|a_{\mu}\right|<\varrho} \log \frac{\left|a_{\mu}\right|}{\varrho}-\sum_{0<\mid b_{\nu}<\varrho} \log \frac{b_{\nu} \mid}{\varrho}-\lambda \log \varrho
\end{aligned}
$$

Hier ist $\lambda$ eine geeignete ganze Zahl derart, daß

$$
c_{j}=\lim _{z \rightarrow 0} z^{-i} w(z)
$$

eine endliche von null verschiedene komplexe Zahl ist.

Im Hinblick auf die Definition der $a$-Stelle einer pseudoanalytischen Funktion $w$ modulo dem erzeugenden Paar $(F, G)$ hat man die Schmiegungsfunktion $m(r, a)$ und die Anzahlfunktion $N(r, a)$ wie folgt festzulegen:

$$
\begin{gathered}
m(r, a)=\frac{1}{2 \pi} \int_{0}^{2 \pi} \log ^{+}+\frac{1}{\mid\left(w\left(r e^{i \vartheta}\right)-\alpha\left(r e^{i \vartheta}\right)\right.} d \vartheta \quad(|a|<\infty, * \alpha(z) \equiv a), \\
m(r, \infty)=\frac{1}{2 \pi} \int_{0}^{2 . \pi} \log +\left|w\left(r \epsilon^{i \vartheta}\right)\right| d \vartheta \\
N(r, a)=\int_{0}^{r} \frac{n(t, a)-n(0, a)}{t} d t+n(0, a) \log r .
\end{gathered}
$$

Dadei bezeichnet $n(r, a)$ für endliches $a$ die in ihrer Vielfachheit gezählten Nullstellen von $w(z)-\alpha(z) \quad(* \alpha(z) \equiv a)$ bzw. die entsprechend ihrer Vielfachheit gezählten Pole von $w$ für unendliches $a$. Anstelle von $m(r, \infty)$ und $N(r, \infty)$ werden auch die Bezeichnungen $m(r, w)$ und $N(r, w)$ benutzt.

Mit diesen Begriffen erhält die Jensensche Formel die Gestalt

$$
m(r, \infty)+N(r, \infty)=m(r, 0) \div N(r, 0) \div h(r, 0),
$$

wenn man $\lambda=n(0,0)-n(0, \infty)$ beachtet und

$$
h(r, 0)=\log \left|c_{\lambda}\right|-\operatorname{Re}\left\{\frac{1}{2 \pi i} \int_{\xi<\varrho}\left[a(\zeta)+b(\zeta) \frac{\overline{w(\zeta)}}{w(\zeta)}\right] \frac{d \zeta d \bar{\zeta}}{\zeta}\right\}
$$

setzt. Wegen Bedingungen (i) und (ii) gilt 


$$
|h(r, 0)| \leqq|\log | c_{\lambda}||+\frac{M M_{1}}{\Delta}
$$

Aus der mit Rücksicht auf die Abschätzungen

$$
\left|\log ^{+}\right| w(z)-\alpha(z)\left|-\log ^{+}\right| w(z)|| \leqq \log ^{+}|\alpha(z)| \div \log 2
$$

und

$$
\log ^{+}|\alpha(z)| \leqq \log ^{+}|a|+\log ^{+} M,
$$

geltenden Ungleichung

$$
|m(r, w)-m(r, w-\alpha)| \leqq \log ^{+}|a|+\log ^{-} M+\log ^{2} 2
$$

folgt mit der für $w-\alpha$ geschriebenen Beziehung (6) wegen der Übereinstimmung der Pole von $w$ mit denen von $w-\alpha$

Der erste Hauptsatz. Für eine in $|z|<R \leqq \infty$ bis auf Pole pseudoanalytische Funktion $w$ und für jede endliche komplexe Zahl a gilt

$$
m(r, \infty)+N(r, \infty)=m(r, a)+N(r, a)+h(r, a)
$$

mit

$$
|h(r, a)| \leqq|\log | c_{\lambda}(a)||+\log ^{+}|a|+\log ^{+} M+\frac{M M_{1}}{\Delta} \div \log 2
$$

Hier bezeichnet $c_{\lambda}(a)$ den (5) entsprechenden Wert für die Funktion $w-\alpha$.

Wie im klassischen Fall wird

$$
T(r, w)=m(r, \infty)+N(r, \infty)
$$

als charakteristische Funktion von $w$ bezeichnet. Aus der PoissonJensenschen Formel läßt sich mit der von Nevanlinna gegebenen Methode (vgl. [7], S. 13) die wichtige Ungleichung

$$
T(t, w) \leqq T(r, w)+\frac{2 M M_{1}}{\Delta} \quad(0 \leqq t \leqq r<R)
$$

ableiten. Genügt eine Funktion einer Ungleichung von der Form (7), so wird sie als fastmonoton bezeichnet.

3. Die logarithmische Methode von Nevanlinna. Zweiter Hauptsatz. Aus der zweiten Gleichung von (4) folgt

$$
m\left(r, \frac{\dot{w}}{w}\right) \leqq m\left(r, \frac{w_{z}}{w}\right)+m\left(r, \frac{F_{z}}{F}\right)+m\left(r, \frac{G_{z}}{G}\right)+\log \div \frac{M^{2}}{1} \div 2 \log 2 .
$$

Durch Differentiation der Poisson-Jensenschen Formel nach $z$ erhält man für ein $z$, das keine Nullstelle und kein Pol von $w$ ist. 


$$
\begin{aligned}
\frac{w_{z}(z)}{w(z)}= & \frac{1}{\pi i} \int_{|\zeta|=\varrho} \log |w(\zeta)| \frac{d \zeta}{(\zeta-z)^{2}} \\
& +\frac{1}{2 \pi i} \int_{|\zeta|<\varrho} \frac{w_{\bar{\zeta}}(\zeta)}{w(\zeta)} \frac{d \zeta d \bar{\zeta}}{(\zeta-z)^{2}}+\frac{\varrho^{2}}{2 \pi i} \int_{|\zeta|<\varrho} \frac{w_{\bar{\zeta}}(\zeta)}{w(\zeta)} \frac{d \zeta d \bar{\zeta}}{\left(\varrho^{2}-\bar{z} \zeta\right)^{2}} \\
& +\sum_{\left|a_{\mu}\right|<\varrho}\left[\frac{\overline{a_{\mu}}}{\varrho^{2}-\overline{a_{\mu}} z}-\frac{1}{a_{\mu}-z}\right]-\sum_{\left|b_{v}\right|<\varrho}\left[\frac{\overline{b_{v}}}{\varrho^{2}-\bar{b}_{\nu} z}-\frac{1}{b_{v}-z}\right] .
\end{aligned}
$$

Zum Beweis bezeichne $2 \pi i g(z)$ das durch

$$
g(z)=\frac{1}{2 \pi i} \int_{|\zeta|<\varrho} \frac{w_{\bar{\zeta}}(\zeta)}{w(\zeta)} \frac{d \zeta d \bar{\zeta}}{\zeta-z}+\frac{\bar{z}}{2 \pi i} \int_{|\zeta|<\varrho} \frac{w_{\bar{\zeta}}(\zeta)}{w(\zeta)} \frac{d \zeta d \bar{\zeta}}{\varrho^{2}-\bar{z} \zeta}
$$

dargestellte Gebietsintegral in der Poisson-Jensenschen Formel. Man beachte außerdem

$$
\frac{\partial}{\partial z} \operatorname{Re} g(z)=\frac{1}{2}\left[g_{z}(z)+\overline{g_{\tilde{z}}(z)}\right] .
$$

Mit Rücksicht auf die Hölderstetigkeit von

$$
w_{\bar{z}} w^{-1}=a+b \bar{w} w^{-1}
$$

in von Nullstellen und Polen von $w$ verschiedenen Punkten existiert die als Cauchysches Hauptwertintegral gegebene Ableitung

$$
g_{z}(z)=\frac{1}{2 \pi i} \int_{|\zeta|<\varrho} \frac{w_{\bar{\zeta}}(\zeta)}{w(\zeta)} \frac{d \zeta d \bar{\zeta}}{(\zeta-z)^{2}}=\frac{1}{2 \pi i} \int_{|\zeta|<\varrho}\left[\frac{w_{\bar{\zeta}}(\zeta)}{w(\zeta)}-\frac{w_{\bar{z}}(z)}{w(z)}\right] \frac{d \zeta d \bar{\zeta}}{(\zeta-z)^{2}},
$$

dessen Hauptwert durch das auf der rechten Seite stehende uneigentliche Integral gegeben ist. Außerdem gilt

$$
g_{\bar{z}}(z)=\frac{w_{\bar{z}}(z)}{w(z)}+\frac{\varrho^{2}}{2 \pi i} \int_{|\bar{\xi}|<Q} \frac{w_{\bar{\zeta}}(\zeta)}{w(\zeta)} \frac{d \zeta d \bar{\zeta}}{\left(\varrho^{2}-\bar{z} \zeta\right)^{2}} .
$$

Die Differentiation der übrigen Glieder in der Poisson-Jensenschen Formel erfolgt wie in [7].

Zur Abschätzung der beiden vorangegangenen Gebietsintegrale für von Nullstellen und Polen von $w$ verschiedene $z$ mit $|z|<\varrho$ entfernt man aus $|z| \leqq \varrho$ alle

$$
n(\varrho)=n(\varrho, 0)+n(\varrho, \infty)
$$

Nullstellen und Pole von $w$ durch offene, untereinander punktfremde Kreise mit positivem Radius $d=d(\varrho) / n(\varrho)$ und den auszuschließenden 
Stellen als Mittelpunkten. Dabei sei $d(\varrho)$ so klein gewählt, daß auch die entsprechenden Kreise mit den Radien $3 d$ noch zueinander disjunkt sind und im Hinblick auf (3) für jede auszuschließende Stelle $z_{0}$ die Abschätzung

$$
\left|\frac{\dot{w}(z)}{w(z)}\right| \leqq \frac{2|n|}{\left|z-z_{0}\right|} \quad\left(\left|z-z_{0}\right|<3 d\right)
$$

richtig ist. In dem so verbleibenden Bereich $B(d, \varrho)$ erfüllt $\bar{w} w^{-1}$ eine Lipschitzbedingung mit der Konstante

$$
L(\varrho)=\sup _{\xi, z \in B(d, g)} \frac{1}{|\zeta-z|}\left|\frac{\overline{w(\zeta)}}{w(\zeta)}-\frac{\overline{w(z)}}{w(z)}\right| .
$$

Da wegen (4)

$$
\left|\frac{w_{\bar{\zeta}}(\zeta)}{w(\zeta)}-\frac{w_{\bar{z}}(z)}{w(z)}\right| \leqq|a(\zeta)-a(z)|+|b(\zeta)-b(z)|+|b(z)|\left|\frac{\overline{w(\zeta)}}{w(\zeta)}-\frac{\overline{w(z)}}{w(z)}\right|
$$

gilt und da mit Hilfe der für ein Gebiet $D$ und beliebiges komplexes $z$ geltenden Ungleichung von E. Schmidt [12]

$$
\left[\frac{1}{2 \pi} \int_{D} \frac{d \xi d \eta}{|\zeta-z|}\right]^{2} \leqq \frac{1}{\pi} \int_{D} d \xi d \eta
$$

für $z$ aus $B(d, \varrho)$ die Abschätzung

$$
\left|\frac{1}{2 \pi i} \int_{B(d, \varrho)}\left[\frac{\overline{w(\zeta)}}{w(\zeta)}-\frac{\overline{w(z)}}{w(z)}\right] \frac{d \zeta d \bar{\zeta}}{(\zeta-z)^{2}}\right| \leqq 2 \varrho L(\varrho)
$$

und für einen der ausgeschlossenen Kreise und $z$ aus $B(2 d, \varrho)$

$$
\left|\frac{1}{2 \pi i} \int_{\left|\zeta-z_{i}\right|<d}\left[\frac{\overline{w(\zeta)}}{w(\zeta)}-\frac{\overline{w(z)}}{w(z)}\right] \frac{d \zeta d \bar{\zeta}}{(\zeta-z)^{2}}\right| \leqq 2
$$

folgt, erhält man insgesamt für $z$ aus $B(2 d, \varrho)$

$$
\left|g_{2}(z)\right| \leqq M_{4}(\varrho) \div \frac{2 M^{2}}{4}[\varrho L(\varrho)+n(\varrho)]
$$

Für den zweiten durch ein Flächenintegral gegebenen Summanden in (9) findet man die Schranke

$$
\frac{M M_{1}}{\Delta} \frac{\varrho}{(\varrho-|z|)^{2}} .
$$


Beide Abschätzungen sind bis auf die Größe $L(\varrho)$ ron der gleichen Güte, wie die der nach Nevanlinna für die übrigen Summanden in (9) geltenden Schranken. Zusammengefaßt erhält man für $\approx$ aus $B(2 d, \varrho)$

$$
\begin{array}{r}
\left|\frac{w_{z}(z)}{w(z)}\right| \\
\leqq \frac{\varrho}{(\varrho-|z|)^{2}}\left[2 m(\varrho, w)+2 m\left(\varrho, \frac{1}{w}\right)+v_{\varrho}(z: w)+v_{\varrho}\left(z, \frac{1}{w}\right)+\frac{M M_{1}}{\Delta}\right] \\
+M_{4}(\varrho)+\frac{2 M^{2}}{1}[\varrho L(\varrho)+n(\varrho)]
\end{array}
$$

mit

$$
v_{\varrho}(z, w)=\sum_{\left|b_{\nu}\right|<\varrho}\left|\frac{\varrho^{2}-\overline{b_{\nu}} z}{\varrho\left(z-b_{\nu}\right)}\right|, \quad v_{\varrho}\left(z, \frac{1}{\omega}\right)=\sum_{a_{\mu}<\varrho}\left|\frac{\varrho^{2}-\overline{a_{\mu}} z}{\varrho\left(z-a_{\mu}\right)}\right| .
$$

Aus (11) ergibt sich eine Schranke für $m\left(r, w_{z} w^{-1}\right)$, wenn der Kreis $|z|=r$ ganz in $B(2 d, \varrho)$ liegt. Daß $m\left(r, \dot{w} w^{-1}\right)$ für diese $r$ eine gleiche obere Schranke mit veränderten Koeffizienten besitzt, folgt mit Rücksicht auf die für die Funktionen $F$ und $G$ geschriebene Beziehung (9), nach der $\left|F_{z}(z) F^{-1}(z)\right|$ und $\left|G_{z}(z) G^{-1}(z)\right|$ durch

$$
\left[2|\log M|+\frac{M M_{1}}{\Delta}\right] \frac{\varrho}{(\varrho-|z|)^{2}} \div 2 \frac{M^{3}}{\Delta^{2}} M_{2}(\varrho) \div M_{4}(\varrho)
$$

nach oben abgeschätzt werden können.

Ist $r<\varrho$ ein oben nicht zugelassener Wert, so zerlege man $|z|=r$ in die beiden Teilmengen

$$
\Gamma_{r}=\{|z|=r\} \cap B(3 d, \varrho), \quad \Gamma_{r}^{\prime}=\{\mid z=r\}-B(3 d, \varrho)
$$

und entsprechend das $m\left(r, \dot{w} w^{-1}\right)$ darstellende Integral in die Summe zweier Integrale. Die Abschätzung des Integrals über $\Gamma_{r}$ erfolgt wie oben mit Hilfe von (8) und (11). Zur Untersuchung des Integrals längs $\Gamma_{r}^{\prime}$ genügt es, einen der endlich vielen Teilbögen ron $\Gamma_{r}^{\prime}$ zu betrachten, etwa den in $\left|z-z_{0}\right|<3 d$ liegenden Bogen ron $z^{\prime}=i$, und dort (10) zu beachten.

Sei $z_{0}^{\prime}$ der für hinreichend großes $r(2 d<r)$ eindeutig bestimmte, in $\left|z-z_{0}\right|<2 d$ gelegene Punkt von $|z|=r$. der den kleinsten Abstand von $z_{0}$ hat, so gilt wegen (10)

$$
\left|\frac{\dot{w}(z)}{w(z)}\right| \leqq \frac{4|n|}{\left|z-z_{0}^{\prime}\right|} \quad\left(|z|=r, \quad ; z-z_{0},<3 d\right)
$$

und mit $\arg z=\vartheta, \arg z_{0}=\vartheta_{0}$ für diese $:$-Werte 


$$
\log ^{+}\left|\frac{\dot{w}(z)}{w(z)}\right| \leqq \log ^{+}|n|+\log ^{+} \frac{1}{2 r}+\log \frac{1}{\sin \frac{\vartheta-\vartheta_{0}}{2} \mid}+\log 8
$$

Da die Länge des in $\left|z-z_{0}\right|<3 d$ gelegenen Bogens $\gamma_{0}$ von $|z|=r$ nicht kleiner als $2 d$ und nicht größer als $6 \pi d$ sein kann, folgt aus der Ungleichung

$$
-\frac{2}{\pi} \int_{0}^{\varphi_{0} / 2} \log \sin \varphi d \varphi \leqq \frac{\varphi_{0}}{\pi}\left[\log \frac{\pi}{\varphi_{0}}+1\right] \quad\left(d \leqq r \varphi_{0} \leqq 3 \pi d\right)
$$

mit Rücksicht darauf, daß die Summe der geeignet gewählten $p_{0}$-Werte nicht größer als $\pi$ ist und daß

$$
\sum_{\varphi_{0}} \frac{\varphi_{0}}{\pi} \log \frac{\pi}{\varphi_{0}} \leqq \frac{3 d n(\varrho)}{r} \log \frac{\pi r}{d}=3 \frac{d(\varrho)}{r}\left[\log \frac{r}{d(\varrho)}-\log n(\varrho)+\log \pi\right]
$$

gilt, die Abschätzung

$\frac{1}{2 \pi} \int_{\Gamma_{r}^{\prime}} \log ^{+}\left|\frac{\dot{w}(z)}{w(z)}\right| d \vartheta \leqq\left[1+3 \frac{d(\varrho)}{r}\right] \log ^{+} n(\varrho)+\log ^{-} \frac{1}{r}-O(1) \quad(r \rightarrow R)$.

Die Größe $d(\varrho) r^{-1}(r<\varrho)$ strebt mit gegen $R$ wachsendem $r$ möglicherweise gegen null, in jedem Fall bleibt sie beschränkt, da $d(\varrho)$ mit wachsendem $\varrho$ nur kleiner werden kann. Auch in dem Fall der oben nicht zugelassenen $r$-Werte ist also eine Abschätzung von der gleichen Art für $m\left(r, \dot{w} w^{-1}\right)$ gewonnen, wie sie für zulässige, aus (11) folgt.

Durch Wahl eines $r^{\prime}$ mit $\varrho<r^{\prime}<R$ und der Festlegung von $\varrho$ durch

$$
\varrho=r+\frac{r}{r^{\prime}} \frac{r^{\prime}-r}{2+T\left(r^{\prime}, w\right)}
$$

erhält man, da noch $L(\varrho) \leqq L\left(r^{\prime}\right)$, für jedes $r<r^{\prime}$

$$
m\left(r, \frac{\dot{w}}{w}\right) \leqq c_{1} \log ^{+} T\left(r^{\prime}, w\right)+\log +L\left(r^{\prime}\right)+c_{2} \tau\left(r^{\prime}\right)-c_{3} \log \frac{1}{r^{\prime}-r}+c_{4}
$$

mit positiven Konstanten $c_{k}(k=1,2,3,4)$.

Ist die Ordnung von $w$ endlich, so folgt mit

$$
r^{\prime}= \begin{cases}2 r, & R=\infty \\ \frac{r+R}{2}, & R<\infty\end{cases}
$$


und der Bezeichnung $L\left(r^{\prime}\right)=L(r, 0)$ für alle $r$ unmittelbar

$$
m\left(r, \frac{\dot{w}}{w}\right) \leqq O(\tau(r))+\log ^{+} L(r, 0) .
$$

Im Fall unendlicher Ordnung von $w$, wenn also

$$
\varlimsup_{r \rightarrow R} \frac{\log T(r, w)}{\tau(r)}=\infty,
$$

wird folgende Erweiterung des Satzes von Borel angewandt.

Hilfssatz (Borel). (1) Die in $0 \leqq r_{0} \leqq r<\infty$ nichtnegative, stetige, mit nichtnegativem $T_{0}$ fastmonotone Funktion $T$ erfüllt für jedes beliebige, feste $k>1$ außerhalb einer Menge $E$ endlichen Maßes die Ungleichung

$$
T\left(r+\frac{1}{T(r)+T_{0}}\right)<k\left[T(r)+T_{0}\right] .
$$

(2) Ist $T$ eine in $0 \leqq r_{0} \leqq r<R<\infty$ gegebene Funktion mit den gleichen Eigenschaften wie in (1), so gilt ausserhalb einer Menge $E$, für die

$$
-\int_{E} d \log (R-r)
$$

endlich ist, für jedes feste $k>1$ die Ungleichung

$$
T\left(r+\frac{R-r}{T(r)+T_{0}+1}\right)<k T(r)+(k+1) T_{0} .
$$

Wählt man nun mit (siehe (7))

$$
r^{\prime}=\left\{\begin{array}{cc}
r+\left[T_{0}+\log +T(r, w)\right]^{-1}, & R=\infty \\
r+\frac{2 M M_{1}}{1+T_{0}+\log ^{+} T(r, w)}, & R<\infty
\end{array}\right.
$$

und schreibt wieder $L\left(r^{\prime}, 0\right)$ für $L\left(r^{\prime}\right)$, so folgt durch Anwendung des Borelschen Satzes auf $\log ^{+} T(r, w)$ der

Hilfssatz über die logarithmische $(F, G)$-Ableitung. Für eine in $|z|<R \leqq+\infty$ pseudoanalytische Funktion gilt außerhalb einer Menge $E$, für die

$$
\int_{E} d \tau(r)
$$


endlich ist, die Ungleichung

$$
m\left(r, \frac{\dot{w}}{w}\right) \leqq O(\log T(r, w))+O(\tau(r))+\log r L(r, 0) .
$$

Im Fall endlicher Ordnung von $w$ ist die Menge $E$ leer.

Um nun den zweiten Hauptsatz herzuleiten, setzt man für $q \geqq 2$ voneinander verschiedene endliche komplexe Zahlen $a_{1}, a_{2}, \ldots, a_{q}$

$$
f(z)=\sum_{k=1}^{q}\left[w(z)-\alpha_{k}(z)\right]^{-1} \quad\left({ }_{*} \alpha_{k}(z) \equiv a_{k}, k=1,2, \ldots, q\right) .
$$

Ist dann

$$
0<\delta_{1} \leqq\left|a_{k}-a_{l}\right| \quad(1 \leqq k<l \leqq q)
$$

so gilt

$$
0<\delta \leqq\left|\alpha_{k}(z)-\alpha_{l}(z)\right| \quad\left(1 \leqq k<l \leqq q, \quad \delta=\frac{\Delta}{M} \delta_{1}\right) .
$$

Mit Hilfe des entsprechenden auf Littlewood zurückgehenden Nevanlinnaschen Beweises folgt

$$
\sum_{k=1}^{q} m\left(r, a_{k}\right) \leqq m(r, f)+O(1) .
$$

Zur Abschätzung von $m(r, f)$ nach oben beachtet man die Ungleichung

$$
|f(z)| \leqq \frac{1}{|\dot{w}(z)|} \sum_{k=1}^{q} \frac{|\dot{w}(z)|}{\left|w(z)-\alpha_{k}(z)\right|},
$$

nach der

$$
m(r, f) \leqq m\left(r, \frac{1}{\dot{w}}\right)+\sum_{k=1}^{q} m\left(r, \frac{\dot{w}}{w-\alpha_{k}}\right)+\log q .
$$

Im Hinblick auf die Bedingungen (ii) an das erzeugende Paar $(F, G)$ ist $w$ nach Bers (vgl. [2], S. 40 ff.) bezüglich eines Nachfolgepaares $\left(F_{1}, G_{1}\right)$ von $(F, G)$ pseudoanalytisch und es gilt

$$
(\dot{w})_{\bar{z}}=a \dot{w}-B \overline{\dot{w}} .
$$

Die mit Rücksicht auf die Endlichkeit des zweiten Integrals in (ii) für $\dot{w}$ ausgesprochene B€ziehung (6) lautet

$$
T(r, \dot{w})=m(r, \dot{w})+N(r, \dot{w})=m\left(r, \frac{1}{\dot{w}}\right)+N\left(r, \frac{1}{\dot{w}}\right)+O(1) .
$$

Wie im analytischen Fall mißt die Anzahlfunktion 


$$
N_{1}(r)=N\left(r, \frac{1}{\dot{w}}\right)-N(r, \dot{w})+2 N(r, w)
$$

alle Stellen von $u$ mit um eins verminderter Vielfachheit, so daß nur die mehrfachen Stellen von $w$ einen Beitrag zu $N_{1}(r)$ liefern. Da noch

$$
m(r, \dot{w}) \leqq m(r, w)-m\left(r, \frac{\dot{w}}{w}\right), \quad T(r, w)=m(r, w)+N(r, w),
$$

folgt mit $a_{q+1}=\propto$ aus (12) und (13)

$$
\begin{aligned}
& \sum_{k=1}^{q+1} m\left(r, a_{k}\right) \\
& \leqq 2 T(r, u)-N_{1}(r)+m\left(r, \frac{\dot{w}}{w}\right)+\sum_{k=1}^{q} m\left(r, \frac{\dot{w}}{w-\alpha_{k}}\right)+O(1) .
\end{aligned}
$$

Bezeichnet $L\left(r, a_{k}\right)$ die $L(r, 0)$ entsprechende Lipschitzkonstante für die Funktion $u-\alpha_{i}$ und setzt man noch $a_{0}=0$, so folgt aus dem auf die Funktionen $w-a_{k}$ angewandten Hilfssatz über die logarithmische Ableitung der zweite Nevanlinnache Hauptsatz.

Der zweite Hauptsatz. Für eine in $|z|<R$ nichtkonstante pseudoanalytische Funktion gilt mit $q \geqq 0$ endlichen oder unendlichen, voneinander verschiederen komplexen Zahlen $a_{k}(k=1,2, \ldots, q)$ und $a_{0}=0$

$$
\begin{aligned}
& (q-2) T(r, w) \\
& \leqq \sum_{k=1} N\left(r, w_{k}\right)-N_{1}(r)+\sum_{\substack{k=0 \\
a_{k}<\infty}}^{q} \log +L\left(r, a_{k}\right)+O(\log T(r, w))+O(\tau(r))
\end{aligned}
$$

außerhalb einer Henge $E$ von $r$-Werten endlichen $\tau$-Haßes. Im Fall endicher Oidnung von $w$ ist diese Ausnahmemenge leer.

Macht man über die $L\left(r, a_{k}\right)$ eine geeignete Wachstumsvoraussetzung, so lassen sich die üblichen Folgerungen aus dieser Ungleichung ziehen. Bekanntlich [4] gilt der zweite Hauptsatz auch für pseudoanalytische Funktionen in der gewohnten Form ohne die von den Lipschitzkonstanten herrührenden Glieder. Hier scheint die potentialtheoretische der logarithmischen Methode überlegen zu sein. Im analytischen Fall liefert zwar die erstgenannte Methode im Unterschied zur zweiten eine scharfe Ungleichung, jedoch wird auf beiden Wegen noch die gleiche Funktionenklasse erfaßt. Bei der Untersuchung von pseudoanalytischen Funktionen mit Hilfe der potentialtheoretischen Methode entfallen alle die Hölderstetigkeit der erzeugenden Funktionen und ihrer partiellen Ableitungen nach $\bar{z}$ betreffenden Voraussetzungen, so daß sie von vornherein eine größere Klasse von Funktionen zuläßt. 


\section{Literatur}

[1] Begehr, H.: Zur Wertverteilung approximativ analytischer Funktionen. Arch. Math. (Basel) 23, 1972, S. 41-49.

[2] Bers, L.: Theory of pseudo-analytic functions. - [Vorlesungsausarbeitung.] New York University, Institute for Mathematics and Mechanics, New York, 1953.

[3] Dinghas, A.: Vorlesungen über Funktionentheorie. - Die Grundlehren der mathematischen Wissenschaften 110. Springer-Verlag, Berlin / Göttingen / Heidelberg, 1961.

[4] Habetha, K.: Über die Werteverteilung pseudoanalytischer Funktionen. Ann. Acad. Sci. Fennicæe A. I. 406, 1967.

[5] AF HäLlströм, G.: Wertverteilungssätze pseudomeromorpher Funktionen. · Acta Acarl. Åbo. Math. Phys. 21: 9, 1958.

[6] Hayman, W. K.: Meromorphic functions. - Oxford mathematical monographs. Clarendon Press, Oxford, 1964.

[7] Nevanlinna, R.: Zur Theorie der meromorphen Funktionen. - Acta Math. 46,1925, S. $1-99$.

[8] - $\gg-$ Le théorème de Picard-Borel et la théorie des fonctions méromorphes. . Collection dir monographies sur la théorie des fonctions. GauthierVillars et C Éditeurs, Paris, 1929.

[9] - - Inalytic functions. - Die Grundlehren der mathematischen Wissenschaften 162. Springer-Verlag, Berlin / Heidelberg / New York, 1970.

[10] NirexberG, L.: On nonlinear elliptic partial differential equations and Hölder continuity. - Comm. Pure Appl. Math. 6, 1953, S. 103-156.

[11] Ponpeid, D.: Sur une classe de fonctions d'une variable complexe. - Rend. Cire. Mat. Palemo 33, 1912, S. 108-113.

[12] Schmdt, E.: Bemerkung zur Potentialtheorie. - Mathematische Abhandlungen Hermann Amandus Schwarz zu seinem fünfzigjährigen Doktorjubiläum am 6. Angust 1914 gewidmet von Freunden und Schülern. S. 365-383. Terlag ron Julius Springer, Berlin, 1914.

[13] VEKT. I. N.: Generalized analytic functions. - International series of monographs on pure and applied mathematics 25. Pergamon Press, Oxford / London/New York/Paris, 1962.

[14] Wrтtich, H.: Neuere Untersuchungen über eindeutige analytische Funktionen. - [Zweite, korrigierte Aufiage.] Ergebnisse der Mathematik und ihrer Grenzgebiete [Neue Folge] 8. Springer-Verlag, Berlin/Heidelberg / New York, 1968.

Freie Universität Berlin

I. Mathematisches Institut

D-1000 Berlin 33

Deutschland 